\title{
A Novel Low-profile Log-periodic Ultra Wideband Feed for the Dual-reflector Antenna of US-SKA
}

\author{
Rikard Olsson', Per-Simon Kildal ${ }^{2}$ and Sander Weinreb ${ }^{3}$ \\ ${ }^{1.2}$ Chalmers University of Technology, Göteborg, Sweden \\ ${ }^{3}$ California Institute of Technology, Pasadena, California \\ ${ }^{1}$ E-mail: rikard.olsson@elmagn.chalmers.se \\ ${ }^{2}$ E-mail: simon@elmagn.chalmers.se \\ ${ }^{3}$ E-mail: sweinreb@caltech.edu
}

\begin{abstract}
We present a novel low-profile log-periodic ultra wideband (UWB) dipole antenna referred to as the Chalmers Feed. The simulated results presented shows that the antenna has low input reflection coefficient, low cross-polarization, constant beam width and constant phase center position over more than a decade bandwidth. The antenna can be enclosed in a volume of $0.5 \lambda_{L} \cdot 0.5 \lambda_{L} \cdot 0.15 \lambda_{L}$ size, where $\lambda_{L}$ is the wavelength at the lowest frequency. The groundplane needs to be somewhat larger. The antenna is intended to feed a dual-reflector antenna proposed by the US-SKA consortium.
\end{abstract}

\section{INTRODUCTION}

The square kilometer array (SKA) is a proposed radio telescope of one square kilometer aperture for use between $500 \mathrm{MHz}$ and $34 \mathrm{GHz}$. The US-SKA is a US proposal for SKA consisting of a large number of $12 \mathrm{~m}$ offset dual-reflector antennas with broadband feeds. The Allen telescope array (ATA) is a similar but smaller radio telescope under construction in the USA consisting of 350 dual-reflector antennas of $6 \mathrm{~m}$ aperture diameter. A wideband log-periodic feed for ATA has been designed by Greg Engargiola [1]. David Ericsson [4] and the present author in his master thesis made some studies on this wideband ATA feed. In the studies it was shown that the ATA feed has good impedance match but exhibits variation of phase center with frequency and significant back lobes. In addition it is quite long which makes cryogenic cooling (for noise reduction) of the feed very difficult. In Rikard Olsson's master thesis possible ways to improve the ATA feed and the surrounding cooling Dewar were studied. In the master thesis we also proposed a new low-profile log-periodic dipole comb feed for US-SKA at frequencies up to 12 $\mathrm{GHz}$. This feed is referred to as the Chalmers feed. In the present paper we present computeraided modeling of a metal strip realization of the Chalmers feed that may remove the disadvantages of the ATA feed. In the future we plan to continue the optimization of the Chalmers feed including dual polarization, inclusion of a dielectric substrate, and a finite ground plane followed by construction and testing of a prototype. In the present study the method of moments is used as implemented in the commercial code WIPL-D. For characterizing the feed performance the reflector is assumed to be a body of revolution (BOR), see [3] and [4]. Thereby, the feed can be characterized by the classical fecd efficiency and its sub-efficiencies, see [3] and [4]. The feed efficiency is the aperture efficiency of a rotationally symmetric Cassagrain antenna when the effects of center blockage and subreflector diffraction are neglected. It includes losses due to spill-over, higher order $\varphi$-variation in the radiation pattern, cross-polarization, nonuniform aperture distribution and phase errors. In table 1 we see an explanation some of the different sub-efficiencies used to characterize the feed performance. These sub-efficiencies can be found, in more detailed form, in [3] and [4].

The Chalmers feed is protected by a patent application [5]. 


\section{II .DESCRIPTION OF FEED AND ANALYSIS}

The goal when designing the Chalmers feed was to overcome the problems with the ATA feed, in particular its size and its varying phase center location. The basic geometry was therefore chosen to be two parallel dipoles separated by $D=0.5$ lbegin $\{$ math $\}$ llambda lend math $\}$ and located above a ground plane, a classical feed geometry known to give equal $\mathrm{E}$ - and $\mathrm{H}$-plane patterns and lock the phase center to the ground-plane. The large bandwidth is obtained by adding more dipole pairs and scaling them logarithmically in size relative to each other. We chose to realize the dipoles as cascaded folded dipoles with one common input port, by means of a two-strip transmission line that could be printed on a circuit board. This makes the feed cheap to manufacture. Figure 1 shows a drawing of the Chalmers feed with the definition of its dimensions. Figure 1 also illustrates how the cascaded dipoles are connected together using the two-strip transmission line. Figure 2 shows a drawing of what the feed would look like if it was used in dual linear polarization and printed on a dielectric circuit board.

The dipole arms and transmission lines of the feed analyzed in the present paper are all realized by perfectly conducting strips. In the analysis there is so far no dielectric supporting the strips and the ground plane is assumed to be infinite

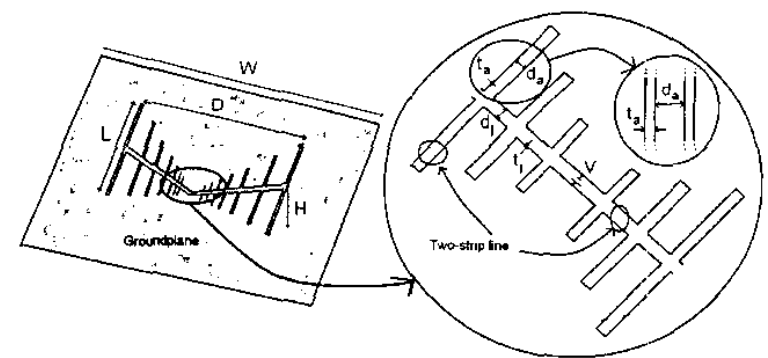

Fig. 1. Drawing of the low-profile log-periodic Chalmers feed with illustration of the central feed part at which the feed voltage $V$ is located between the two strips of the two-strip transmission line. This voltage $\mathrm{V}$ excites both log-periodic parts of the feed. The outer strip of the outermost folded dipoles are short circuited as shown.

Fig. 2. Drawing of the low-profile log-periodic Chalmers feed used in two perpendicular linear polarizations. In this paper however only one linear polarization is so far studied.

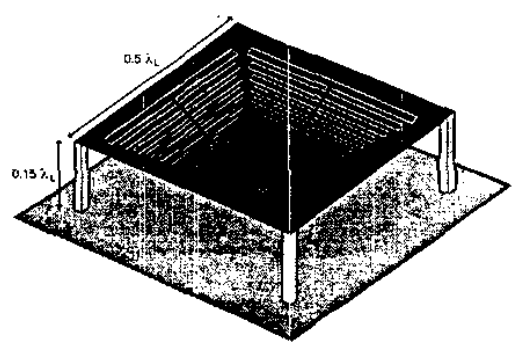

III. RESULTS

The computed performance of the present version of the feed between 1 and $12 \mathrm{GHz}$ is shown in figure 3 and 4 . To the left in figure 3 we see the radiation patterns at 23 linearly separated 
frequency points between 1 and $12 \mathrm{GHz}$. The beam width is close to constant. The feed efficiency, shown to the right in figure 3 , is excellent over the entire band with rather small fluctuations. The efficiency due to mismatch is around $-0.7 \mathrm{~dB}$ corresponding to an input reflection coefficient better than $-10 \mathrm{~dB}$ at most frequencies and better than $-6 \mathrm{~dB}$ at all frequencies as shown in figure 4. An interesting figure of merit is the total efficiency. For the ATA feed this is $-3.7 \mathrm{~dB}$ [3] (for a subtended half angle of $42 \mathrm{deg}$ towards the subreflector) at the worst frequencies. For the new Chalmers feed it is $-2.7 \mathrm{~dB}$ (for a subtended half angle of 50 deg towards the subreflector) at the worst frequencies. Note that the ATA feed has been analyzed as a dual-polarized feed while the Chalmers feed only includes one polarization. This could account for part of the difference in total efficiency.One disadvantage with the Chalmers feed is that the beam is rather wide. We therefore made an investigation of how the feed efficiency is affected when we change the subtended half angle towards the subreflector. The results are shown in figure 5 . As we can see the Chalmers feed has its highest feed efficiency at $50 \mathrm{deg}$. Realizing a dual reflector geometry of the kind proposed for US-SKA for this subtended angle could be difficult. In the ATA project a reflector designed for a subtended half angle of 42 deg has been used. Fortunately we see that the Chalmers feed also works well for $42 \mathrm{deg}$ subtended angle, which indicates that it also could be used in a similar reflector geometry as the ATA feed Changing from $50 \mathrm{deg}$ to $42 \mathrm{deg}$ subtended angle actually only reduces the total feed efficiency by $0.2 \mathrm{~dB}$.

TABLE I

EXPLANATION OF THE EFFICIENCIES USED TO CHARACTERIZE THE FEED PERFORMANCE

\begin{tabular}{lcl} 
Name & Notation & \multicolumn{1}{c}{ Reason for efficiency reduction } \\
\hline $\begin{array}{l}\text { Total efficiency } \\
\text { Feed efficiency }\end{array}$ & $e_{t o r}$ & $\begin{array}{l}\text { Product of feed efficiency and reflection efficiency. } \\
\text { Efficiency when feeding a rotationally including all } \\
\text { contributions except reflection efficiency and center } \\
\text { blockage. }\end{array}$ \\
Spill-over efficiency & $e_{s p}$ & $\begin{array}{l}\text { Power lost in the part of the radiation field that doesn't hit } \\
\text { the reflector. }\end{array}$ \\
Reflection efficiency & $e_{r e f}$ & $\begin{array}{l}\text { Power lost in reflections due to the mismatch at the input } \\
\text { port. }\end{array}$
\end{tabular}

\section{CONCLUSION}

A novel low-profile log-periodic folded dipole feed has been presented when realized as metal strips supported by a dielectric circuit board. The impedance characteristics are acceptable over a very wide band from 1 to $12 \mathrm{GHz}$ with return loss better than $-10 \mathrm{~dB}$ at most frequencies and better than $-6 \mathrm{~dB}$ at all frequencies. We expect that this can be improved by further work. The Chalmers feed illuminates a dual reflector antenna with 50 deg subtended half angle towards the subreflector with low spillover and high aperture efficiency (also called feed efficiency). The feed can also be used for smaller subtended half angles down to 40 deg without significant reduction in feed efficiency. The feed looks to be а уегу strong candidate for the US-SKA project.

\section{REFERENCES}

[1] G. Engargiola, "Non-planar log-periodic antenna feed for integration with a cryogenic microwave amplifier", Proceedings of IEEE Antennas and propagation society international symposium in Columbus, page 140-143, June 2002

[2] P-S. Kildal, Foundations of Antennas - A unified approach, Studentlitteratur, 2000, also available at amazaon.com

[3] P-S. Kildal, "Factorization of the feed efficiency of paraboloids and Cassegrain antennas", IEEE Transactions on Antennas and Propagation, Volume: 33 Issue: 8, Aug 1985 
[4] D. Ericsson, P-S. Kildal and S. Weinreb, "Study of efficiencies and phase centers of broadband $\log$-periodic feeds for large offset dual-reflector antennas using formulas for bodies of revolution (BOR1 extraction)", Proceedings of IEEE Antennas and propagation society international symposium in Columbus, June 2003.

[5] P-S. Kildal, "Broadband multi-dipole antenna with frequency-independent radiation characteristics", Swedish Patent Application No. 0302175-5, 7 August 2003
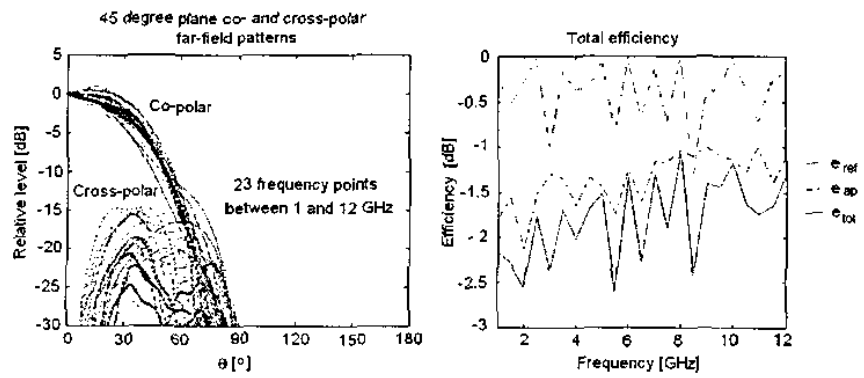

Fig. 3. Calculated Co- and cross-polar 45 degree plane relative far-field patterns (left) and feed efficiencies (right) of the Chalmers feed.

Fig. 4. The reflection coefficient seen on a transmission line of

$100 \Omega$ characteristic impedance.

A least square fit has been

used to generate the curve.

The circles indicate actual

simulation points.
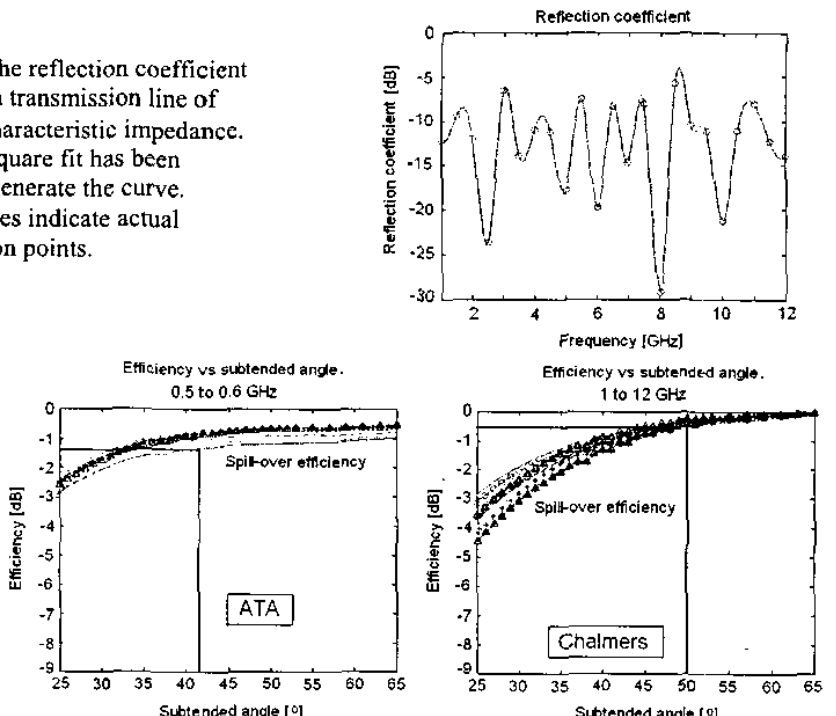

Fig. 5. The spill-over efficiency (left) and the feed efficiency (right) versus the subtended half angle towards the sub-reflector for the Chalmers feed. The feed efficiency is an approximation of the aperture efficiency of the dual reflector antenna. 